\title{
Design of robust controller for STATCOM applied to large induction motor using normalized coprime factorization approach
}

\begin{abstract}
Between the different robust $\mathrm{H}$-infinity methods to design the controller for FACTS, loop shaping is known to be one of the effective and feasible methods. This research presents an investigation on $\mathrm{H}$-infinity loop shaping procedure through normalized coprime factorization (NCF) for STATCOM installed at terminals of a large induction motor. The dynamic behaviors of induction motor are analyzed while the uncertainty of system parameters and STATCOM parameters are considered and will be compared with conventional PI controller. Simulation results prove that NCF method has better dynamic response and is more robust than PI controller against variation of system parameters and STATCOM parameters, so in a specified operating point even the system goes to instability.
\end{abstract}

Keyword: STATCOM; Robust control; Normalized coprime factorization; Uncertainty modeling 